\title{
Cytotoxic Effect of Disulfiram/Copper On Human Cervical Cancer Cell Lines and LGR5-Positive Cancer Stem-Like Cells
}

\section{Hao-Zhe Cao}

Xi'an Jiaotong University Medical School

Peng-Sheng Zheng ( $\nabla$ zpsheng@mail.xjtu.edu.cn )

Xi'an Jiaotong University Medical School

\section{Wen-Ting Yang}

Xi'an Jiaotong University Medical School

\section{Research Article}

Keywords: LGR5, disulfiram, copper, cervical cancer, cancer stem cell

Posted Date: December 29th, 2021

DOI: https://doi.org/10.21203/rs.3.rs-1122434/v1

License: (c) (1) This work is licensed under a Creative Commons Attribution 4.0 International License.

Read Full License 


\section{Abstract}

Background: Tumor resistance is a global challenge for tumor treatment. Cancer stem cells (CSCs) are the main population of tumor cells for drug resistance. We have reported that high aldehyde dehydrogenase (ALDH) activity represents a functional marker for cervical CSCs. Here we aim at disulfiram (DS), an ALDH inhibitor, that has the potential as a novel treatment to be used for cervical cancer.

Methods: MTT assay, western blot, FCS analysis and sorting, vector construction and transfection, in vivo anti-tumor assays were performed using cervical cancer cell lines SiHa and HeLa. Cell cycle distribution and cell apoptosis were carried out by flow cytometry. The cytotoxicity of DS was detected by MTT assay and a xenograft cervical cancer model.

Results: Disulfiram was cytotoxic to cervical cancer cell lines in a copper (Cu)-dependent manner. Disulfiram/copper (DS/Cu) complex induced deregulation of S-phase and inhibited the expression of stemness marker in cervical cancer cells. DS/Cu caused the death of LGR5-positive cervical cancer cells, a cancer stem-like cell population, which lead to cisplatin resistance in cervical cancer cells. Furthermore, DS/Cu complex had the greater antitumor efficacy on cervical cancer than cisplatin group in vitro and in vivo.

Conclusion: Our findings indicate that the cytotoxicity of DS/Cu complex may be superior to cisplatin because of targeting LGR5-positive cervical cancer stem-like cells in cervical cancer. Thus, the DS/Cu complex may represent a potential therapeutic strategy for cervical cancer patients.

\section{Background}

Cervical cancer is the third most common type of malignant tumor and the fourth leading cause of cancer death among women worldwide [1, 2]. Although HPV testing and Papanicolaou smear screening have brought great benefits to the prevention and early treatment of cervical cancer, the patients with metastatic still face great difficulties in treatment. The five-year survival rate of cervical cancer patients without metastasis was over $80 \%$, but the survival rate of metastasis patients was unfavorable and under $42 \%$ without dramatic improvement for several decades [3]. It is critical to develop a novel treatment in order to increase the overall survival rate of cervical cancer patients.

Disulfiram (DS) has been used as a first-line anti-alcoholism drug in the world for over 60 years [4, 5]. DS is a small molecule with a molecular weight of 296.54. Recently, accumulating evidence demonstrates that DS is involved in inhibiting prostate cancer, lung cancer, breast cancer, liver cancer, ovarian cancer and oesophageal carcinoma cell proliferation [6-13]. DS also potentiates the cytotoxicity of several classical anticancer drugs as well as radiation [14-18]. DS can be rapidly reduced to form two molecules of diethyldithiocarbamate (EtDTC) in serum, which is a strong chelator of transition divalent metal ions [19]. The anticancer effect of DS has been shown to be copper (Cu) dependent [20-22]. DS improves the transport of copper into cancer cells [23]. Copper plays an important role in redox reactions and triggers 
generation of reactive oxygen species (ROS) in the cells [24]. In addition, previous studies reported that the DS/Cu complex is highly cytotoxic to the cancer cells but not the normal cells $[25,26]$. It is noteworthy that the repurposing of DS to cancer treatment has received recent interest.

It has been proposed that disulfiram-loaded immediate and extended release vaginal tablets have the potential to be used for the localised treatment of cervical cancer $[27,28]$. However, these studies showed that the inhibition of cervical cancer cell viability required a high dose of disulfiram and did not involve the addition of copper. We have found that high aldehyde dehydrogenase (ALDH) activity represents both a functional marker for cervical cancer stem cells (CCSCs) and a target for cervical cancer therapies [29]. In addition, our previous studies demonstrated that LGR5 promotes cancer stem cell traits and chemoresistance in cervical cancer [30]. These findings led us to investigate the effect of disulfiram and copper in cervical cancer and CCSCs.

In the present work, we examined the inhibitory effect of disulfiram alone and in combination with copper on cervical cancer cell proliferation and cisplatin(DDP) sensitivity. We also determined that disulfiram/copper complex reduced cervical cancer stem-like cells than cisplatin group in vitro. DS/Cu complex had the greater antitumor efficacy on cervical cancer than cisplatin group in vivo. Our data suggest that disulfiram/copper complex alone or combined with cisplatin is a potential therapeutic strategy for cervical cancer patients.

\section{Methods}

\section{Cell culture}

The cervical cancer cell lines $\mathrm{SiHa}$ and HeLa were cultured in DMEM medium with L-glutamine (Invitrogen, Heidelberg, Germany) supplemented with $10 \%$ fetal bovine serum and $1 \%$ penicillin/streptomycin (both from Millipore, Darmstadt, Germany) in a humidified incubator at $37^{\circ} \mathrm{C}$ and $5 \% \mathrm{CO}_{2}$. Both cell lines were chosen due to their different degrees of sensitivity to DS treatment.

\section{MTT assay}

To account for different growth rates, cervical cancer cells were seeded in triplicates in 96-well plates at densities that provided $70 \%$ confluency after $72 \mathrm{~h}$. Different doses of disulfiram (Sigma), $\mathrm{CuCl}_{2}$ (Sigma) and (or) $10 \mu \mathrm{mol} / \mathrm{L}$ cisplatin (DDP, Teva Parenteral, CA) were used in combination assays as indicated. Cell viability was assessed using the 3-(4,5-Dimethyl-1, 3-thiazol-2-yl)-2,5-diphenyl-2H-tetrazol-3-ium bromide (MTT; Sigma-Aldrich) assay. Following the manufacturer's instructions, $20 \mu \mathrm{l}$ of MTT solution was added to $200 \mu \mathrm{l}$ of the culture media. The plates were then incubated for $4 \mathrm{~h}$ at $37^{\circ} \mathrm{C}$, and the optical density was measured at $490 \mathrm{~nm}$.

\section{Assessment of apoptosis}

Apoptotic status was determined by PE Annexin-V/7-AAD Apoptosis Detection Kit I (559763, BD) using flow cytometry following the manufacturer's instructions. Briefly, $5 \times 10^{5}$ cells were seeded in T25 flasks 
for $24 \mathrm{~h}$ and treated with drugs for a further $48 \mathrm{~h}$. The cells were rinsed with calcium-containing HBSS and detached using EDTA-free trypsin. The detached cells were washed twice with cold PBS and then resuspended in $100 \mu \mathrm{l}$ Binding Buffer at a concentration of $1 \times 10^{6} \mathrm{cells} / \mathrm{ml}$. All cells were incubated with PE Annexin V $(5 \mu \mathrm{l})$ and 7-AAD $(5 \mu \mathrm{l})$ at RT for $15 \mathrm{~min}$ in the dark. The cells were diluted in $400 \mathrm{ml}$ of Binding Buffer and analysed by a FACSCalibur Flow Cytometry (Becton Dickinson, USA). Apoptosis and necrosis were evaluated using FL2 (Annexin V) vs FL3 (7-AAD) plots. The cells stained with Annexin V only were classified as early apoptosis and the Annexin $V$ and 7-AAD double-stained cells were classified as late apoptosis or necrosis.

\section{Western blot}

Cells were lysed in a lysis buffer (50 mM Tris-HCl, pH 7.4; 150 mM NaCl; 2 mM EDTA; 1\% NP-40; and 0.1\% sodium dodecyl sulfate) that contained a protease inhibitor cocktail (Complete Mini; Roche Diagnostics, Branchburg, NJ). The membranes were incubated with antibodies against human Bcl2 (1:200, sc-7382, Santa Cruz Biotechnology), ABCG2 (1:50, sc-58222, Santa Cruz Biotechnology), P53 (1:500, sc-6246, Santa Cruz Biotechnology), P27 ${ }^{\text {Kip } 1}$ (1:500 dilution, sc-528, Santa Cruz Biotechnology) and $\beta$-actin (1:1000 dilution, sc-47778, Santa Cruz Biotechnology) at $4^{\circ} \mathrm{C}$ overnight followed by incubation with horseradish peroxidase-conjugated secondary immunoglobulin G (IgG; Thermo Fisher Scientific, New York, NY). The membranes were briefly incubated with an enhanced chemiluminescence reagent (Millipore, Billerica, Mass) and then visualized on X-ray films [31].

\section{Cell cycle analysis}

Analysis of cell cycle progression was performed using DNA staining. Briefly, cervical cancer cells were plated into T-75 flasks at an initial seeding density of $10^{6}$ cells/flask. After $48 \mathrm{~h}$ treatment, trypsinized cells were washed twice with cold PBS, followed by fixation with ice-cold $70 \%$ ethanol overnight at $4^{\circ} \mathrm{C}$. After washing twice with PBS, the cells were incubated with $50 \mu \mathrm{g} / \mathrm{ml}$ propidium iodide (Sigma-Aldrich, St. Louis, MO, USA) and $10 \mu \mathrm{g} / \mathrm{ml}$ RNaseA (Sigma) for $30 \mathrm{~min}$ at room temperature. The cells were then analyzed using a FACSCalibur (Becton Dickinson, USA) with the FlowJo software (Tree Star Inc., Ashland, USA).

\section{Flow cytometry analysis and FACS isolation of cells}

The ALDH enzymatic activity of the cells was measured using the ALDEFLUOR kit (Stem Cell Technologies, Vancouver, BC, Canada), according to the manufacturer's instructions. The brightly fluorescent ALDH-expressing cells were detected using a FACSCalibur flow cytometer (BD Biosciences). As a negative control, cells were stained under identical conditions after treatment with the specific ALDH inhibitor diethylaminobenzaldehyde (DEAB) [29]. The expression of LGR5 and CD49f in cervical cancer cells was measured using the Alexa Fluor ${ }^{\circledR} 647$ Rat anti-Human LGR5 (N-Terminal) antibody (562903, $\mathrm{BD}$ ) and PE Rat anti-Human CD49f antibody (555736, BD) according to the manufacturer's instructions. The data were analyzed using the CellQuest program (BD Biosciences).

\section{Vector construction and transfection}


Human full-length LGR5 cDNA was amplified by reverse transcription polymerase chain reaction using mRNA extracted from SW620 cells. The primer sequences were designed as follows:

F5'-CTTCTCGAGCTACTTCGGGCACCATGG AC-3'; and

R5'-GCGGGTACCTTAGAGACATGGGACAAA TG-3'.

The LGR5 DNA fragment was subsequently cloned into the Xhol and Smal sites of the pCAG-AcGFP vector (Clontech, Mountain View, CA, USA) to generate the pCAG-AcGFP-LGR5 recombinant plasmid. The LGR5-overexpression vectors were transfected into SiHa and HeLa cells using the Lipofectamine 2000 reagent (Invitrogen, Carlsbad, CA, USA) according to the manufacturer's protocol. The transfected cells were treated with G418 (Calbiochem, La Jolla, CA, USA) for 3 weeks, and drug-resistant colonies were collected, expanded, and identified [30].

In vivo anti-tumor assays

The cervical cancer bearing BALB/c nude mice model was used to investigate the in vivo antitumor efficacy of disulfiram/copper complex. Female BALB/c-nude mice (6-7 weeks old) were obtained from Slac Laboratory Animal Co., Ltd (Shanghai, China) and housed in an SFP room that was maintained at a constant temperature $\left(22^{\circ} \mathrm{C}-25^{\circ} \mathrm{C}\right)$ and humidity $(40-50 \%)$. Flow sorted tumor cells $\left(1 \times 10^{6}\right)$ were resuspended in 200 $\mu \mathrm{l}$ of 1:1 PBS/Matrigel (BD Biosciences) solution and injected into the subcutis of the two flanks on the dorsum of each mouse (eight mice in each group). Drug treatment (DS $30 \mathrm{mg} / \mathrm{kg}, \mathrm{CuCl}_{2}$ $1.5 \mathrm{mg} / \mathrm{kg}$, DDP $5 \mathrm{mg} / \mathrm{kg}, 0.9 \%$ saline) was initiated three days after cell injection and administered via intraperitoneally injection twice weekly. DS (6 mg/ml stock) was prepared as follsixows: $120 \mathrm{mg}$ DS was dissolved in $1 \mathrm{ml}$ DMSO, made up to $10 \mathrm{ml}$ with PEG300 (Selleck), then after mixing was added to $10 \mathrm{ml}$ $\mathrm{H}_{2} \mathrm{O}$, vortexed and filter sterilised. Animals had access to food and water ad libitum. The tumor size was measured using a vernier caliper every week, and the volume was calculated with the following formula: $\mathrm{V}=\left(\right.$ length $\times$ width $\left.^{2}\right) / 2$.

\section{Statistical analysis}

Statistical analyses were performed using the GraphPad Prism 8.00 software (La Jolla, CA, USA). In the comparisons of 2 groups, Student's t-test was used to determine statistical significance. To examine differences among $>2$ groups, non-parametrical Kruskal-Wallis test or one-way analysis of variance (ANOVA) was used to determine statistical significance. Kaplan-Meier survival analysis was performed, and survival curve comparisons were performed using the log-rank (Mantel-Cox) test. $P$ values of $\leq 0.05$ were regarded as statistically significant.

\section{Results}

\subsection{DS and copper produce synergistic cytotoxicity in cervical cancer cell lines}


Initially, cell viability assays were conducted to investigate the sensitivity of a panel of cervical cancer cell lines to disulfiram. SiHa and HeLa cell lines were treated with different concentrations of DS or copper $\left(\mathrm{CuCl}_{2}, \mathrm{Cu}\right)$ alone followed by MTT assay. No difference cell viality was observed both in cervical cancer cell lines until the cells were treated with $10 \mu \mathrm{M}$ disulfiram and $0.01 \mu \mathrm{M} \mathrm{CuCl}_{2}$ alone $(P>0.05)$. However, in the presence of $0.01 \mu \mathrm{M} \mathrm{CuCl}_{2}$, as low as $0.1 \mu \mathrm{M}$ disulfiram showed strong cytotoxicity in SiHa cells and $0.01 \mu \mathrm{M}$ disulfiram in HeLa cells (both at $P<0.001$ ). The addition of $10 \mu \mathrm{M}$ cisplatin significantly improved the cytotoxicity of $\mathrm{DS} / \mathrm{CuCl}_{2}$ complex at concentrations ranged from $0.001 \mu \mathrm{M}$ to $0.1 \mu \mathrm{M} \mathrm{DS}$ in SiHa cells and $0.01 \mu \mathrm{M}$ to $1 \mu \mathrm{M}$ DS in HeLa cells $(P<0.05$, Fig. $1 \mathrm{a}-\mathrm{b})$.

To further investigate the synergistic cytotoxicity of DS/Cu complex, cell viability of SiHa and HeLa cells was measured after treatment with a constant low-dose of $\mathrm{DS} / \mathrm{CuCl}_{2}$ comprared with $10 \mu \mathrm{M}$ DDP alone or in combination for different incubation times. The cytotoxicity of $\mathrm{DS} / \mathrm{CuCl}_{2}$ was significantly stronger than DDP alone at all different time points in both cell lines $(24 \mathrm{~h}, 48 \mathrm{~h}, 72 \mathrm{~h}$; all at $P<0.01)$. DS/ $\mathrm{CuCl}_{2}$ complex combined with DDP showed significantly greater cytotoxicity than $\mathrm{DS} / \mathrm{CuCl}_{2}$ only at the time point of $24 \mathrm{~h}$ in two cancer cell lines $(P<0.01$, Fig. $1 \mathrm{c}-\mathrm{d})$. These dshowedata indicated that $\mathrm{DS} / \mathrm{CuCl}_{2}$ complex alone or combinated with DDP showed produce appreciable cytotoxicity in cervical cancer cells.

\subsection{Disulfiram/copper complex induces apoptosis in cervical cancer cell lines}

The apoptotic status of drug-treated cervical cancer cell lines was examined. SiHa and HeLa cells were treated with $\mathrm{DS} / \mathrm{CuCl}_{2}$ complex $\left(0.1 \mu \mathrm{M} \mathrm{DS}+0.01 \mu \mathrm{M} \mathrm{CuCl}_{2}\right), 10 \mu \mathrm{M} \mathrm{DDP}$ or DS/CuCl 2 plus $10 \mu \mathrm{M} \mathrm{DDP}$ (DS/ $\left.\mathrm{CuCl}_{2} / \mathrm{DDP}\right)$ for $48 \mathrm{~h}$. The apoptotic population was detected by flow cytometric with Annexin V/7AAD staining (Fig. 2a). In comparison with the control or DDP treated groups, a large population of apoptotic cells were detected in $\mathrm{DS} / \mathrm{CuCl}_{2}$ and $\mathrm{DS} / \mathrm{CuCl}_{2} / \mathrm{DDP}$-treated cells, respectively $(P<0.01$, Fig. 2bc). The apoptotic results were also confirmed by western blotting analysis of the expression of some apoptosis-related proteins and resistance protein. DS/ $\mathrm{CuCl}_{2} / \mathrm{DDP}$ induced p53 and p27 protein expression in $\mathrm{SiHa}$ and HeLa cells. DS/ $\mathrm{CuCl}_{2}$ induced p53 and p27 protein expression in HeLa cells. In addition, $\mathrm{DS} / \mathrm{CuCl}_{2}$ inhibited the expression of bcl2 and $\mathrm{ABCG} 2$ protein in two cervical cancer cell lines. The expression of ABCG2 protein in DDP group was significantly higher than $\mathrm{DS} / \mathrm{CuCl}_{2} / \mathrm{DDP}$ group in two cell lines $(P<0.0001$, Fig. 2d-g).

\subsection{Disulfiram/copper complex induces the decrease of S- phase in cervical cancer cells}

To investigate the mechanism by which disulfiram/copper complex inhibits cervical cancer cell proliferation, we analyzed cell cycle status before and after treatment. Exposure of SiHa and HeLa cells to $0.02 \mu \mathrm{M}$ disulfiram plus $0.01 \mu \mathrm{M} \mathrm{CuCl}_{2}$ caused a significant decrease of cells in S-phase relative to control group (both at $P<0.05$ ). Consistently, co-exposure of $\mathrm{SiHa}$ and $\mathrm{HeLa}$ cells to $\mathrm{DS} / \mathrm{CuCl}_{2} / \mathrm{DDP}$ caused a significant decrease of cells in S-phase relative to DDP groups (both at $P<0.05$ ). In addition, 
$\mathrm{DS} / \mathrm{CuCl}_{2}$ treatment led to increased SiHa cells in $\mathrm{G} 2 / \mathrm{M}$ phase relative to control group. $\mathrm{DS} / \mathrm{CuCl}_{2} / \mathrm{DDP}$ treatment led to increased $\mathrm{SiHa}$ and HeLa cells in G2/M phase relative to DDP alone, respectively $(P<$ 0.05 , Fig. 3a-d). The data suggested that disulfiram/copper synergistically inhibited cervical cancer cell proliferation through the decrease of S-phase and G2/M phase arrest.

\subsection{Disulfiram/copper complex inhibits the expression of stemness marker in cervical cancer cells}

It is widely accepted that CSCs are resistant to a wide range of classical cytotoxic anticancer drugs. Our previous study demonstrated that high ALDH activity could represent a functional marker for CCSCs [29]. Disulfiram is a specific inhibitor of ALDH. In this study, we examined the expression of ALDH in DS/CuCl 2 and DDP treated SiHa and HeLa cell lines. After $18 \mathrm{~h}$ exposure to different drugs and following culture in drug-free culture medium for 3 days, a significantly lower percentage of ALDH-positive cells were detected in $0.1 \mu \mathrm{M}$ disulfiram plus $0.01 \mu \mathrm{M} \mathrm{CuCl}_{2}$ treated SiHa cells than control cells $(2.25 \%$ vs $14.70 \%, P<$ 0.0001). In contrast, a significantly higher percentage of ALDH-positive cells were detected in 10 $\mu \mathrm{M}$ DDP treated $\mathrm{SiHa}$ cells than control cells $(36.16 \%$ vs $14.70 \%, P<0.0001)$ and $\mathrm{DS} / \mathrm{CuCl}_{2} / \mathrm{DDP}$ treated cells $(36.16 \%$ vs $4.99 \%, P<0.0001)$, respectively (Fig. $4 a, C)$. Although there was no ALDH expression in natural HeLa cells, a significantly higher percentage of ALDH-positive cells were detected in DDP treated HeLa cells than $\mathrm{DS} / \mathrm{CuCl}_{2}(3.40 \%$ vs $0.00 \%, P<0.0001)$ and $\mathrm{DS} / \mathrm{CuCl}_{2} / \mathrm{DDP}$ treated cells $(3.40 \%$ vs $0.07 \%, P<$ $0.0001)$, respectively (Fig. $4 \mathrm{~b}, \mathrm{~d})$.

We also examined the effect of these compounds on CD49f, another CSC marker. The CD49f expression in $\mathrm{DS} / \mathrm{CuCl}_{2}$ treated $\mathrm{SiHa}$ and HeLa cells was significantly reduced compared with their control cells $(P<$ 0.05). Consistently, the $\mathrm{CD} 49 \mathrm{f}$ expression in $\mathrm{DS} / \mathrm{CuCl}_{2} / \mathrm{DDP}$ treated $\mathrm{SiHa}$ and HeLa cells was significantly reduced compared with DDP treated SiHa and HeLa cells, respectively $(P<0.0001$, Fig. $4 \mathrm{e}-\mathrm{g})$.

\subsection{Disulfiram/copper complex causes the death of LGR5- positive cervical cancer stem-like cells}

In our study, disulfiram/copper complex strongly inhibited ALDH activity in SiHa cell, which may be a successful therapeutic for chemoresistant cervical cancer by successfully targeting chemoresistant CSC via attenuation of ALDH-mediated protection from damage. Although there was no ALDH expression in natural HeLa cells, disulfiram/copper complex obviously induced apoptosis of HeLa cells. Our previous studies indicated LGR5 promotes cancer stem cell traits and chemoresistance in cervical cancer cells [30]. To further confirm whether disulfiram/copper complex were sensitive to the LGR5-positive cells, stable LGR5-overexpressing cells (SiHa-LGR5 and HeLa-LGR5) were established in cervical cancer cell lines. The expression of LGR5 in these cell lines was examined by immunohistochemistry, western blot and flow cytometry (Supplementary Fig. 1). We tested the effects of disulfiram/copper complex and DDP on cervical cancer cells with different expression levels of LGR5. The cell viability was determined using an MTT assay. The cell viability showed time-dependency when these cells treated with different drugs. Both SiHa-LGR5 and HeLa-LGR5 cells were significantly more resistant to DDP than the control cells (SiHa- 
AcGFP and HeLa-AcGFP, $P<0.001)$, but there was no significantly difference between LGR5overexpression cells and control cells treated with $\mathrm{DS} / \mathrm{CuCl}_{2}$ or DS/CuCl $/ 2$ DDP $(P>0.05$, Fig. 5a-b). These results indicated that disulfiram/copper complex produced cytotoxicity in LGR5-positive cervical cancer cells.

To further confirm whether disulfiram/copper complex were sensitive to the LGR5-positive cells, these cells with different drugs for three days and then cultured these cells in regular culture medium for two weeks. In the DDP treated group, the percentage of LGR5 ${ }^{+}$cells expanded from $1.79-23.33 \%$ in the SiHaAcGFP cell population, $81.07-99.03 \%$ in the SiHa-LGR5 cell population, $1.66-21.92 \%$ in the HeLa-AcGFP cell population and $36.27-57.29 \%$ in the HeLa-LGR5 cell population. However, in the disulfiram/copper treated group, the percentage of $\mathrm{LGR}^{+}$cells reduced from $1.79-1.02 \%$ in the SiHa-AcGFP cell population, 81.07-27.74\% in the SiHa-LGR5 cell population, $1.66-0.45 \%$ in the HeLa-AcGFP cell population and $36.27-29.66 \%$ in the HeLa-LGR5 cell population. Additionally, in the combination group of $D S / \mathrm{CuCl}_{2} / \mathrm{DDP}$, the percentage of LGR5 ${ }^{+}$cells is significantly lower than DDP group $(P<0.0001$, Fig. $5 \mathrm{c}-\mathrm{f})$. These data suggested that disulfiram/copper complex could cause the death of LGR5-positive cervical cancer cells which had the ability of resistance to cisplatin treatment.

\subsection{Disulfiram/copper complex inhibits LGR5-positive cervical cancer cells in vivo}

To determine whether DS/Cu can inhibit LGR5-positive cervical cancer stem-like cells in vivo, we used a xenograft model of modified LGR5-positive/negtive SiHa and HeLa cells in BALB/c mice (Fig. 6a). Tumor growth curves are shown in Fig. $6 \mathrm{~b}$ and $\mathrm{c}$. The tumors formed by the $\mathrm{LGR} 5^{+}$cells were larger and grew faster than those formed by the LGR5 ${ }^{-}$cells in each group $(P<0.05)$, but the tumors treated with disulfiram/copper complex were significantly smaller and slower than those treated with DDP or PBS in both modified $\mathrm{SiHa}$ and HeLa cell groups $(P<0.05)$. Upon completion, tumor weight for treatment groups of DS/Cu was smaller than those treated with DDP and PBS groups (Fig. 6d-e). Tumor latency was monitored after the injection of sorted cells into BALB/c mice and was defined the tumor-free duration in the mice. During 8 weeks, the AcGFPLGR5- cell groups treated with DS/Cu caused the higher tumor-free survival rates $(12.5 \%$ for SiHa-AcGFPLGR5- cell group and $25 \%$ for HeLa-AcGFPLGR5- cell group versus $0 \%$ for other groups) than DDP groups and control groups. ( $P<0.05$, Fig. $6 \mathrm{~g}-\mathrm{f})$. Data indicated that treatment with disulfiram/copper complex had the greater efficacy in tumor growth inhibition compared with DDP groups whether in LGR5-positive or LGR5-negtive cervical cancer cells in vivo.

\section{Discussion}

The tumor originates from monoclonal cell and appears to contain a heterogeneous population of tumor cells. According to the cancer stem cell hypothesis, among these heterogeneous cell populations, only a few subpopulation of cancer cells, possess enhanced self-renewal capacity, differentiation potential, 
tumorigenicity and chemoresistance [32]. Our previous studies have demonstrated that high aldehyde dehydrogenase (ALDH) activity may represent a functional marker for cervical CSCs and LGR5 can promote cervical cancer stem cell traits and chemoresistance [29, 30]. Here, we explored to find a drug targeting cervical CSCs for cancer therapies. DS has been shown to be effective against some cancer types in preclinical studies. It has also been observed that the patients who continuously used DS have a lower risk of death from cancer compared with those who stopped using DS at their diagnosis in an epidemiological study [33]. However, little is known about the mechanisms and therapy of disulfiram in cervical cancer. In the present study, we show, for the first time, the cytotoxicity of disulfiram may be superior to cisplatin caused by targeting LGR5-positive cervical cancer stem-like cells in cervical cancer.

In this study, DS with exogenous $\mathrm{Cu}^{2+}$ supplementation exhibited dose-dependent and time-dependent cytotoxicity in cervical cancer cells, but disulfiram alone had no therapeutic effect in votro. Researches reported recently demonstrated that disulfiram/copper treatment eliminated the stem cell-like ALDH ${ }^{+}$ cells pool in non-small cell lung cancer $[34,35]$. We also found disulfiram/copper complex alone or in combination with DDP strongly inhibited ALDH activity in SiHa cells and DDP treated HeLa cells. Although there is no ALDH expression in untreated HeLa cells, disulfiram/copper complex induced more remarkable cell apoptosis and exhibited greater efficacy of tumor growth inhibition on HeLa cells compared with DDP in vitro and in vivo. Previous studies manifested that overexpression of LGR5 promotes cervical cancer cell stemness and chemoresistance. According to this study, disulfiram/copper complex exhibited equivalent effect whether LGR5-positive cervical cancer stem-like cells or LGR5-negtive cells both in vitro and in vivo systems. In other words, disulfiram/copper complex treatment was superior to cisplatin by its ability of eliminating the stem cell-like LGR5 ${ }^{+}$cells pool in HeLa cells.

It has also been reported that DS/Cu treatment causes accumulation of cells in G2 and inhibits DNA synthesis [18]. In our study, the decrease in the proportion of cells in S phase following DS/Cu treatment was accompanied by an increase in the proportion of SiHa cells in $\mathrm{G} 2$ phase. Although the decrease in the proportion of cells in S phase following DS/Cu treatment without increase of G2 phase in HeLa cells, both $\mathrm{SiHa}$ and HeLa cells were corroborated the observation that $\mathrm{DS} / \mathrm{CuCl}_{2} / \mathrm{DDP}$ caused arrest in $\mathrm{G} 2$. In addition, the expression of p53 and p27 was significantly increased in $\mathrm{DS} / \mathrm{CuCl}_{2} / \mathrm{DDP}$ treated $\mathrm{SiHa}$ and HeLa cells $(P<0.05$, Fig. 2). When DNA damage occurs in the G2 phase, the expression levels of p53 and p27 are increased, cells undergo G2/M phase cell cycle arrest, p53 and its downstream gene p27 promotes apoptosis [36-38]. However, in detail, the changes of these genes caused by $\mathrm{DS} / \mathrm{CuCl}_{2}$ are asynchronous in HeLa and SiHa cells, which need further study. Bcl-2, an anti-apoptotic protein, has been reported to regulate the apoptotic pathways and protect against cell death $[39,40]$. The multidrug resistance gene $A B C G 2$ is also an important proliferation-promoting oncogene in cervical cancer [41]. We also observed that $\mathrm{DS} / \mathrm{CuCl}_{2}$ treatment resulted in a marked decrease of bcl-2 and ABCG2 protein expression in $\mathrm{SiHa}$ and HeLa cells, which was superior to that of cisplatin.

Previous studies developed a vaginal tablet of DS to be used in HeLa and Ca-Ski cells for the localised treatment of cervical cancer [28, 42]. This study showed animal experiments provided a basis for 
systemic application of DS. In addition, disulfiram/copper complex could inhibit the expression of stemness marker in cervical cancer cells. It is attractive to consider DS as an independent treatment or adjuvant treatment combined with cisplatin to establish a new chemotherapy protocol for targeting CCSCs. In the combination treatment, DS could suppress the cisplatin-resistant LGR5 ${ }^{+}$stem-like population to cisplatin treatment. At the same time, the cytotoxicity of cisplatin could be increased once combined with DS. However, more research is still needed to further explore the effect of DS on primary tumors from patients.

\section{Conclusion}

Taken together, these results indicate that disulfiram/copper complex is toxic in cervical cancer cell lines in vitro and in vivo by mechanisms including targeting $\mathrm{LGR}^{+}$cancer stem-like cells. We encourage for further clinical trials in cervical cancer with DS, an old, safe and public domain drug [43].

\section{Declarations}

\section{Ethics approval and consent to participate}

The study was approved by the Ethics Committee of The First Affiliated Hospital of Xi'an Jiaotong University.

\section{Consent for publication}

Not applicable.

\section{Availability of data and materials}

The datasets used and/or analyzed during the current study are available from the corresponding author on reasonable request.

\section{Competing interests}

The authors declare that they have no conflicts of interest.

\section{Funding}

This work was supported by a grant Dr. Wen-Ting Yang from the Fundamental Research Funds for the Central Universities (xzy012020064) and a grant awarded to Prof. Peng-Sheng Zheng from the National Natural Science Foundation of China (No. 81472728 )

\section{Authors' contributions}

Peng-Sheng Zheng and Hao-Zhe CAO designed the work. Hao-Zhe CAO collected and integrated the data, prepared the manuscript. Wen-Ting Yang edited and revised the manuscript. All authors read and 
approved the final manuscript.

\section{Acknowledgements}

Not applicable.

\section{References}

1. Brebi MP, Ili GC, López MJ, García MP, Melo AA, Montenegro HS, Leal RP, Guzmán GP, Roa SJ: [Detection and genotyping of human papillomavirus in biopsies of uterine cervical adenocarcinoma]. Revista medica de Chile 2009, 137(3):377-382.

2. Jemal A, Bray F, Center MM, Ferlay J, Forman DJCCJC: Global cancerstatistics. 2011, 6(2):169-190.

3. Carneiro SR, Fagundes MDA, Rosário PDJOD, Neves LMT, Pinheiro MDCNJPO: Five-year survival and associated factors in women treated for cervical cancer at a reference hospital in the Brazilian Amazon. 2017, 12(11):e0187579.

4. Cvek B: Nonprofit drugs as the salvation of the world's healthcare systems: the case of Antabuse (disulfiram). Drug Discov Today 2012, 17(9-10):409-412.

5. Skinner MD, Lahmek P, Pham H, Aubin H-J: Disulfiram Efficacy in the Treatment of Alcohol Dependence: A Meta-Analysis. Plos One 2014, 9(2).

6. Iljin K, Ketola K, Vainio P, Halonen P, Kohonen P, Fey V, Grafström RC, Perälä M, Kallioniemi O: HighThroughput Cell-Based Screening of 4910 Known Drugs and Drug-like Small Molecules Identifies Disulfiram as an Inhibitor of Prostate Cancer Cell Growth. 2009, 15(19):6070-6078.

7. Duan X, Xiao J, Yin Q, Zhang Z, Yu H, Mao S, Li YJN: Multi-targeted inhibition of tumor growth and lung metastasis by redox-sensitive shell crosslinked micelles loading disulfiram. 2014, 25(12):125102.

8. Liu P, Wang Z, Sarah B, Vinodh K, Erebi TP, Jiang W, Irache JM, Tang JZ, Stephen B, Armesilla ALJO: Liposome encapsulated Disulfiram inhibits NFKB pathway and targets breast cancer stem cells in vitro and in vivo. 2014, 5(17):7471-7485.

9. Wang Z, Tan J, Mcconville C, Kannappan V, Tawari PE, Brown J, Ding J, Armesilla AL, Irache JM, Mei QBJNNB et al: Poly lactic-co-glycolic acid controlled delivery of Disulfiram to target liver cancer stemlike cells. 2016:S1549963416301083.

10. He H, Markoutsa E, Li J, Xu PJAB: Repurposing Disulfiram for Cancer Therapy via Targeted Nanotechnology through Enhanced Tumor Mass Penetration and Disassembly. 2017:S1742706117307894.

11. Butcher K, Kannappan V, Kilari RS, Morris MR, McConville C, Armesilla AL, Wang W: Investigation of the key chemical structures involved in the anticancer activity of disulfiram in A549 non-small cell lung cancer cell line. BMC Cancer 2018, 18(1):753.

12. Wiggins HL, Wymant JM, Solfa F, Hiscox SE, Taylor KM, Westwell AD, Jones AT: Disulfiram-induced cytotoxicity and endo-lysosomal sequestration of zinc in breast cancer cells. Biochem Pharmacol 
2015, 93(3):332-342.

13. Jivan R, Peres J, Damelin LH, Wadee R, Veale RB, Prince S, Mavri-Damelin D: Disulfiram with or without metformin inhibits oesophageal squamous cell carcinoma in vivo. Cancer Letters 2018, 417:1-10.

14. Rae C, Tesson M, Babich JW, Boyd M, Sorensen A, Mairs RJ: The role of copper in disulfiram-induced toxicity and radiosensitization of cancer cells. Journal of nuclear medicine: official publication, Society of Nuclear Medicine 2013, 54(6):953-960.

15. Yip NC, Fombon IS, Liu P, Brown S, Kannappan V, Armesilla AL, Xu B, Cassidy J, Darling JL, Wang W: Disulfiram modulated ROS-MAPK and NFKB pathways and targeted breast cancer cells with cancer stem cell-like properties. Br J Cancer 2011, 104(10):1564-1574.

16. Inoue K, Fukunaga M, Yamasawa K: Effect of disulfiram and its reduced metabolite, diethyldithiocarbamate on aldehyde dehydrogenase of human erythrocytes. Life Sciences 1982, 30(5):419424.

17. Zhang H, Chen D, Ringler J, Chen W, Cui QC, Ethier SP, Dou QP, Wu G: Disulfiram treatment facilitates phosphoinositide 3-kinase inhibition in human breast cancer cells in vitro and in vivo. Cancer Res 2010, 70(10):3996-4004.

18. Tesson M, Anselmi G, Bell C, Mairs R: Cell cycle specific radiosensitisation by the disulfiram and copper complex. Oncotarget 2017, 8(39):65900-65916.

19. Buac D, Schmitt S, Ventro G, Kona FR, Dou QP: Dithiocarbamate-based coordination compounds as potent proteasome inhibitors in human cancer cells. Mini Rev Med Chem 2012, 12(12):1193-1201.

20. Bhadhprasit W, Kodama H, Fujisawa C, Hiroki T, Ogawa E: Effect of copper and disulfiram combination therapy on the macular mouse, a model of Menkes disease. Journal of Trace Elements in Medicine and Biology 2012, 26(2-3):105-108.

21. Cen DZ, Brayton D, Shahandeh B, Meyskens FL, Farmer PJ: Disulfiram facilitates intracellular Cu uptake and induces apoptosis in human melanoma cells. Journal of Medicinal Chemistry 2004, 47(27):6914-6920.

22. Safi R, Nelson ER, Chitneni SK, Franz KJ, George DJ, Zalutsky MR, McDonnell DP: Copper signaling axis as a target for prostate cancer therapeutics. Cancer Res 2014, 74(20):5819-5831.

23. Nagai M, Vo NH, Ogawa LS, Chimmanamada D, Inoue T, Chu J, Beaudette-Zlatanova BC, Lu RZ, Blackman RK, Barsoum J et al: The oncology drug elesclomol selectively transports copper to the mitochondria to induce oxidative stress in cancer cells. Free Radical Biology and Medicine 2012, 52(10):2142-2150.

24. Morrison BW, Doudican NA, Patel KR, Orlow SJ: Disulfiram induces copper-dependent stimulation of reactive oxygen species and activation of the extrinsic apoptotic pathway in melanoma. Melanoma Res 2010, 20(1):11-20.

25. Yang Y, Li M, Sun X, Zhou C, Wang Y, Wang L, Chen L, Liang Z, Zhu L, Yang H: The selective cytotoxicity of DSF-Cu attributes to the biomechanical properties and cytoskeleton rearrangements in 
the normal and cancerous nasopharyngeal epithelial cells. International Journal of Biochemistry \& Cell Biology 2017, 84:96-108.

26. Chen D, Cui QC, Yang H, Dou QP: Disulfiram, a clinically used anti-alcoholism drug and copperbinding agent, induces apoptotic cell death in breast cancer cultures and xenografts via inhibition of the proteasome activity. Cancer Res 2006, 66(21):10425-10433.

27. Boyd P, Major I, Wang W, McConville C: Development of disulfiram-loaded vaginal rings for the localised treatment of cervical cancer. Eur J Pharm Biopharm 2014, 88(3):945-953.

28. Abidin IZ, Rezoagli E, Simonassi-Paiva B, Fehrenbach GW, Masterson K, Pogue R, Cao Z, Rowan N, Murphy EJ, Major I: A Bilayer Vaginal Tablet for the Localized Delivery of Disulfiram and 5Fluorouracil to the Cervix. Pharmaceutics 2020, 12(12).

29. Liu SY, Zheng PS: High aldehyde dehydrogenase activity identifies cancer stem cells in human cervical cancer. Oncotarget 2013, 4(12):2462-2475.

30. Cao HZ, Liu XF, Yang WT, Chen Q, Zheng PS: LGR5 promotes cancer stem cell traits and chemoresistance in cervical cancer. Cell Death Dis 2017, 8(9):e3039.

31. Chen Q, Cao HZ, Zheng PS: LGR5 promotes the proliferation and tumor formation of cervical cancer cells through the Wnt/ $\beta$-catenin signaling pathway. Oncotarget 2014, 5(19):9092-9105.

32. Fulawka L, Donizy P, Halon A: Cancer stem cells-the current status of an old concept: literature review and clinical approaches. Biol Res 2014, 47(1):66.

33. Skrott Z, Mistrik M, Andersen KK, Friis S, Majera D, Gursky J, Ozdian T, Bartkova J, Turi Z, Moudry P et al: Alcohol-abuse drug disulfiram targets cancer via p97 segregase adaptor NPL4. Nature 2017, 552(7684):194-199.

34. Jin N, Zhu X, Cheng F, Zhang L: Disulfiram/copper targets stem cell-like ALDH(+) population of multiple myeloma by inhibition of ALDH1A1 and Hedgehog pathway. J Cell Biochem 2018, 119(8):6882-6893.

35. Liu X, Wang L, Cui W, Yuan X, Lin L, Cao Q, Wang N, Li Y, Guo W, Zhang X et al: Targeting ALDH1A1 by disulfiram/copper complex inhibits non-small cell lung cancer recurrence driven by ALDH-positive cancer stem cells. Oncotarget 2016, 7(36):58516-58530.

36. Fridman JS, Lowe SW: Control of apoptosis by p53. Oncogene 2003, 22(56):9030-9040.

37. Zhang $Y, N a R$, Wang $X$ : LncRNA WT1-AS up-regulates $p 53$ to inhibit the proliferation of cervical squamous carcinoma cells. BMC Cancer 2019, 19(1):1052.

38. Luo YH, Wang C, Xu WT, Zhang Y, Zhang T, Xue H, Li YN, Fu ZR, Wang Y, Jin CH: 18ß-Glycyrrhetinic Acid Has Anti-Cancer Effects via Inducing Apoptosis and G2/M Cell Cycle Arrest, and Inhibiting Migration of A549 Lung Cancer Cells. Onco Targets Ther 2021, 14:5131-5144.

39. Fleischer B, Schulze-Bergkamen H, Schuchmann M, Weber A, Biesterfeld S, Müller M, Krammer PH, Galle PR: Mcl-1 is an anti-apoptotic factor for human hepatocellular carcinoma. Int J Oncol 2006, 28(1):25-32. 
40. Nagireddy PKR, Kumar D, Kommalapati VK, Pedapati RK, Kojja V, Tangutur AD, Kantevari S: 9-Ethynyl noscapine induces $\mathrm{G} 2 / \mathrm{M}$ arrest and apoptosis by disrupting tubulin polymerization in cervical cancer. Drug Dev Res 2021.

41. Ding $M$, Zhang H, Liu L, Liang R: Effect of NOS1 regulating ABCG2 expression on proliferation and apoptosis of cervical cancer cells. Oncol Lett 2019, 17(2):1531-1536.

42. Baffoe CS, Nguyen N, Boyd P, Wang W, Morris M, McConville C: Disulfiram-loaded immediate and extended release vaginal tablets for the localised treatment of cervical cancer. $J$ Pharm Pharmacol 2015, 67(2):189-198.

43. Cvek B: Nonprofit drugs as the salvation of the world's healthcare systems: the case of Antabuse (disulfiram). Drug Discov Today 2012, 17(9-10):409-412.

\section{Figures}

a

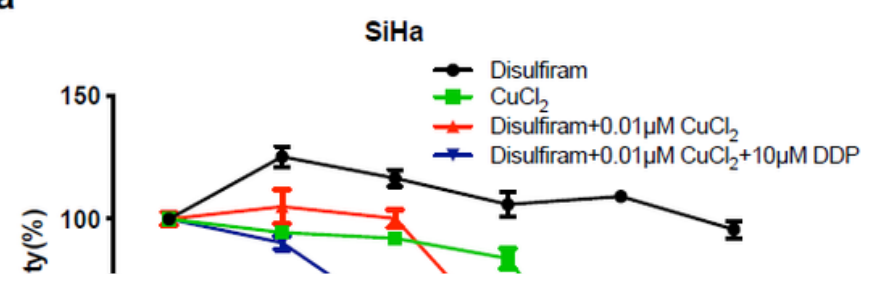

b

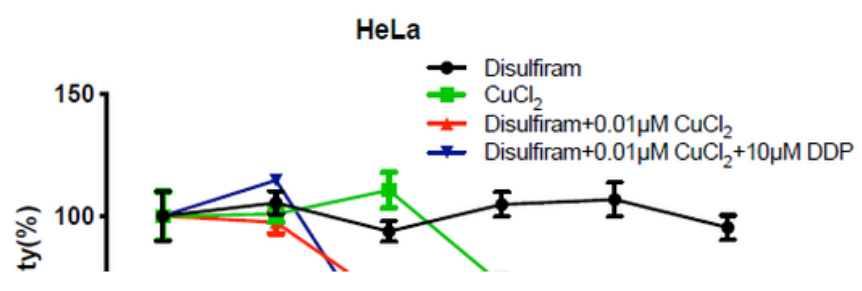

Figure 1

Inhibition of cervical cancer cell proliferation by disulfiram/copper complex. (a-b) SiHa and HeLa cells were treated with different concentrations of disulfiram and $\mathrm{CuCl}_{2}$ alone or in combination with or without $10 \mu \mathrm{M}$ DDP for $24 \mathrm{~h}$, after which their cell viabilities were determined by the MTT assay. (c-d) SiHa and HeLa cells were treated at different time points $(0,24,48$, and $72 \mathrm{~h})$ with a constant low-dose of 
disulfiram/copper complex compared with DDP alone or in combination, after which their cell viabilities were determined by the MTT assay. Data represent mean \pm S.D. of triplicate experiments.

\section{Figure 2}

Disulfiram/copper complex induces apoptosis in cervical cancer cell lines. (a) SiHa and HeLa cells were exposed to $10 \mu \mathrm{M}$ DDP, $0.1 \mu \mathrm{M}$ disulfiram plus $0.01 \mu \mathrm{M} \mathrm{CuCl}_{2}\left(\mathrm{DS} / \mathrm{CuCl}_{2}\right.$ ) or $\mathrm{DS} / \mathrm{CuCl}_{2}$ plus $10 \mu \mathrm{M}$ DDP (DS/ $\left.\mathrm{CuCl}_{2} / \mathrm{DDP}\right)$ in combination for $48 \mathrm{~h}$. The drug-treated and control cells were stained with 7-AAD and PE-Annexin $V$ and then analysed by flow cytometry. (b-c) The percentage of different cell populations identified by 7-AAD/Annexin $V$ assay. (d-e) The expression of apoptosis-related proteins and resistance protein by western blot analysis. (f-g) The relative expression levels of bcl2 and ABCG2 were analysed by

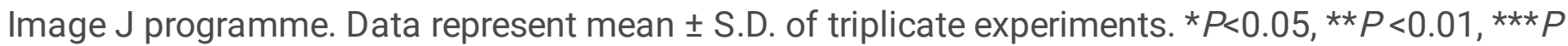
$<0.001, \star \star \star \star P<0.0001$.

\section{Figure 3}

Effect of disulfiram/copper complex on cervical cancer cells through the cell cycle. (a-b) SiHa and HeLa cells were treated with $0.02 \mu \mathrm{M}$ disulfiram plus $0.01 \mu \mathrm{M} \mathrm{CuCl}_{2}, 10 \mu \mathrm{M}$ DDP alone or their combination as indicated for 2 days and then followed by cell cycle analysis. (c-d) DS/CuCl 2 and $\mathrm{DS} / \mathrm{CuCl}_{2} / \mathrm{DDP}$ treatment induced the decrease of cervical cancer cells in S-phase compared with the control and DDP groups, respectively. Data represent mean \pm S.D. of triplicate experiments. ${ }^{\star} P<0.05$.

\section{Figure 4}

\section{Disulfiram/copper complex inhibits the expression of stemness markers in cervical cancer cell lines.} ALDH enzyme activity (a-b) and CD49f expression (e) in two cervical cancer cell lines were analyzed by flow cytometry after $18 \mathrm{~h}$ exposure to different drug and following culture in drug-free culture medium for 3 days. As a negative control for ALDH, the cells were treated with the specific ALDH inhibitor DEAB. The gated cells represent the ALDH-positive cells. Disulfiram/copper complex inhibited the expression of ALDH (c-d) and CD49f (f-g) in SiHa and HeLa cells. Data represent mean \pm S.D. of triplicate experiments. $\star P<0.05, \star \star P<0.01, * \star \star P<0.001, * \star \star \star P<0.0001$.

\section{Figure 5}


Disulfiram/copper complex causes the death of LGR5-positive cervical cancer cells. (a-b) The LGR5overexpressing cervical cancer cells and the control cells were treated with $0.1 \mu \mathrm{M}$ disulfiram plus 0.01 $\mu \mathrm{M} \mathrm{CuCl}_{2}\left(\mathrm{DS} / \mathrm{CuCl}_{2}\right)$ or $10 \mu \mathrm{M}$ DDP or DS/CuCl 2 plus $10 \mu \mathrm{M} \mathrm{DDP}\left(\mathrm{DS} / \mathrm{CuCl}_{2} / \mathrm{DDP}\right)$ for $24 \mathrm{~h}$ and $48 \mathrm{~h}$, after which their cell viabilities were determined by the MTT assay. (c-d) The percentage of LGR5-positive cells in the cervical cancer cell lines was analyzed by flow cytometry after exposure to different drugs for 3 days and following culture in ordinary culture medium for 2 weeks. (e-f) Graphical representation of the statistical analysis of the percentage of LGR5-positive cells in different groups. Data represent mean \pm

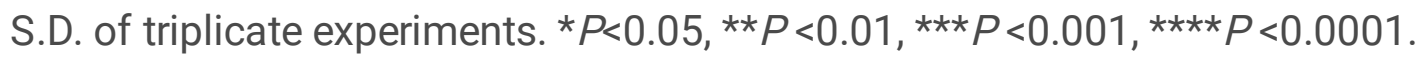

\section{Figure 6}

Disulfiram/copper complex inhibits LGR5-positive cervical cancer cells in vivo. (a) Suppression of tumor growth after disulfiram/copper complex and DDP treatment in female BALB/c-nude mice. (b-c) The volume of xenograft tumors formed by LGR5 ${ }^{\text {LGR5+ }}$ and AcGFPLGR5- cervical cancer cells with different drug delivery was monitored over time. (d-e) At the end of the experiment, the tumor weights were measured. (f-g) Kaplan-Meier plots showing the tumor-free survival after injection. Data represent mean \pm

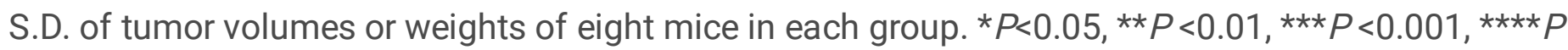
$<0.0001$.

\section{Supplementary Files}

This is a list of supplementary files associated with this preprint. Click to download.

- SupplementaryFigure1.pdf 